\title{
Phase behavior of monomeric mixtures and polymer solutions with soft interaction potentials
}

\author{
C. M. Wijmans ${ }^{\text {a) }}$ and B. Smit \\ Department of Chemical Engineering, University of Amsterdam, Nieuwe Achtergracht 166, \\ 1018 WV Amsterdam, The Netherlands \\ R. D. Groot \\ Unilever Research Vlaardingen, Olivier van Noortlaan 120, 3133 AT Vlaardingen, The Netherlands
}

(Received 6 December 2000; accepted 14 February 2001)

\begin{abstract}
We present Gibbs ensemble Monte Carlo simulations of monomer-solvent and polymer-solvent mixtures with soft interaction potentials, that are used in dissipative particle dynamics simulations. From the simulated phase behavior of the monomer-solvent mixtures one can derive an effective Flory-Huggins $\chi$-parameter as a function of the particle interaction potential. We show that this $\chi$-parameter agrees very well with the free energy difference between a monomer surrounded by solvent particles, and a solvent particle surrounded by solvent particles. We develop a new "identity change" Monte Carlo move to equilibrate the polymer-solvent mixtures. In this move a polymer chain from one box is exchanged with an equal number of solvent particles from the other box. At realistic densities this new move offers a large computational advantage over the convential insertion method for a polymer chain using a configurational bias Monte Carlo algorithm. The new algorithm is demonstrated for polymer-solvent mixtures with a chain length of up to 150 segments. Significant differences are found between the simulated polymer-solvent phase behavior and results predicted by mean-field theory. Finally, we fit a master-equation to the simulated binodal curves at different chain lengths. This function is used to make a quantitative comparison between the simulations and experimental data for the phase equilibrium of the polystyrene-methylcyclohexane system. (C) 2001 American Institute of Physics. [DOI: 10.1063/1.1362298]
\end{abstract}

\section{INTRODUCTION}

In this paper we investigate the phase behavior of a binary mixture of soft spheres similar to those used in the dissipative particle dynamics (DPD) technique. This technique was introduced a few years ago by Hoogerbrugge and Koelman $^{1,2}$ to simulate the hydrodynamic behavior of fluids. It was later extended to polymers by introducing bead-andspring type particles. ${ }^{3-6}$ Español and Warren ${ }^{7}$ showed how the noise and friction terms in the DPD method should be chosen to satisfy the fluctuation-dissipation theorem. When this condition is satisfied the model corresponds to a Hamiltonian system. Because of the coarse-grained character of the simulation model, in which several molecular groups can be incorporated into one simulation particle, this technique is well suited for simulations on a mesoscopic scale. For example, Groot et al. ${ }^{8,9}$ used this method to study block copolymer mesophase formation. Jury et al. ${ }^{10}$ used DPD simulations of a minimal amphiphile model to study amphiphilic mesophases, and Venturoli and Smit ${ }^{11}$ simulated the selfassembly of membranes with more realistic molecular parameters.

The aim of this paper is to gain a better insight into the phase behavior of monomer-monomer and polymer-solvent mixtures in the DPD model. Groot and Warren ${ }^{5}$ were the first authors to study the demixing of DPD particles by conduct-

${ }^{a}$ Electronic mail: chris@its.chem.uva.nl ing DPD simulations. They assumed that their simulations could be interpreted on the basis of the Flory-Huggins theory for polymer solutions. This then makes it possible to use solubility parameters to determine the interaction parameters in a (mesoscopic) DPD simulation. However, this procedure may introduce non-negligible errors due to the meanfield approximation that is made in Flory-Huggins theory. Whether such errors are indeed large can be tested by simulating the (full) binodal curve. This is a computationally very demanding task, though, especially near the critical point. However, as the DPD model corresponds to a Hamiltonian system, one can use different simulation methods to generate the equilibrium configurations of the soft sphere model. Over the past years the advance of molecular simulation techniques has produced the tools we require to simulate efficiently the demixing of a polymer solution.

In the late 1980's Panagiotopolous introduced the Gibbs ensemble Monte Carlo simulation methodology, ${ }^{12-14}$ which describes the equilibrium between two phases without explicitly taking the interface between both phases into account. Initially developed for the liquid-vapor equilibrium of a one-component system, this method was soon extended to binary mixtures. ${ }^{13}$ Another major advancement was made by combining the Gibbs ensemble method with the CBMC (configurational bias Monte Carlo) algorithm, ${ }^{15,16}$ which is a very efficient way of generating configurations of chain molecules. Thus it became possible to investigate the phase behavior of macromolecular systems. 
For monomer mixtures it has been shown that one can determine the phase behavior more efficiently using Gibbs ensemble techniques than using DPD simulations. ${ }^{17}$ In this paper we apply the Gibbs ensemble method both to binary mixtures of soft spheres (monomers) and to bead-and-spring polymers in a monomeric solvent. In the next two sections we first define the model that is used in the simulations. In Sec. IV we then present results for monomer mixtures. The simulated data are compared with predictions from meanfield theory, assuming that the interparticle interactions can be described by an effective Flory-Huggins $\chi$ parameter. The thermodynamic significance of this parameter is explored in some more detail. Finally, we investigate the phase behavior of polymer-solvent systems. In order to simulate these systems we need to develop a new Monte Carlo move to equilibrate the system. The simulated coexistence curves are then compared with curves predicted by Flory-Huggins theory using the $\chi$ parameters derived for monomeric systems.

\section{MODEL}

We consider a particle $n$ of type $i$ and a particle $m$ of type $j$. These two particles interact via a soft repulsive potential

$$
U^{\mathrm{rep}}\left(r_{n m}\right)=\left\{\begin{array}{l}
\frac{1}{2} a_{i j}\left(1-r_{n m}\right)^{2} \quad \text { for } 0 \leqslant r_{n m} \leqslant r_{c}=1 \\
0 \quad \text { for } r_{n m} \geqslant 1
\end{array},\right.
$$

where $r_{n m}$ is the distance between particles $n$ and $m ; a_{i j}$ is the repulsion parameter that defines the interaction between particles of type $i$ and $j$; and $r_{c}$ is the cut-off distance for the interaction force, which defines our unit of length: $r_{c}=1$. We use $a_{i i}=a_{j j}>0$ and $a_{i j}=a_{i i}+\Delta a(\Delta a>0)$. If we are, for example, modeling water, and we assume that one water molecule maps onto one DPD particle, the repulsion parameter must be set to $a_{i i}=25$ to get the correct compressibility. ${ }^{5}$ One can of course map a different number of water molecules onto one DPD particle. If one chooses to map three water molecules onto a DPD particle, one needs a value of $a_{i i}=75$ to match the compressibility of water. Most results presented in this paper will use $a_{i i}=25$ and a small number of simulations will be presented using $a_{i i}=75$ to explore the effect of the $a_{i i}$ parameter.

Polymer chains are formed as a linear array of monomers. Neighboring segments are held together by the following bonding potential:

$$
U^{b}(r)=2 r^{2}+r^{4},
$$

where $r$ is the bond length. This potential is slightly steeper than that used in Ref. 5, because we want to impose an upper limit on the maximum bond length, as will be explained in Sec. III A.

The "soft"' character of the particle interaction potential means that bonds can cross. This has important implications for the dynamics of this model. Spenley ${ }^{6}$ showed that a polymer melt behaves in excellent agreement with Rouse theory.
However, one does not expect that the crossability of bonds will have any important effect on the the equilibrium properties of the system.

\section{SIMULATION METHOD}

We consider a Gibbs ensemble, consisting of two subsytems ("boxes") I and II, with a total volume $V=V^{\mathrm{I}}$ $+V^{\mathrm{II}}$. The system contains $N_{S}$ solvent particles and $M$ polymer chains. Each polymer chain consists of $r$ segments (so that the total number of polymer segments is $N_{P}=M r$ ). The total density is defined as $\rho=N / V$, where $N=N_{S}+N_{P}$. We want to calculate the coexistence curve for this system as a function of $\Delta a$.

The Gibbs ensemble simulation technique has been described in detail many times before (see, for example, Ref. 18). We equilibrate the system using the following Monte Carlo moves:

(1) Displacement. A particle is chosen at random and is given a random displacement. The move is then accepted with a probability $P^{a c}=\min (1, \exp (-\beta \Delta U))$, where $\Delta U$ is the energy change due to the displacement and $\beta$ $=1 / k_{B} T$;

(2) particle exchange. One of the two components is selected at random, and one of the two boxes is selected at random. Then a particle of the selected species is chosen at random and transferred to the other box. This move is accepted with a probability

$$
P^{a c}=\min \left(1, \exp \left[\ln \left(\frac{N_{i}^{d} V^{a}}{\left(N_{i}^{a}+1\right) V^{d}}\right)-\beta \Delta U\right]\right),
$$

where $N_{i}^{d}$ and $N_{i}^{a}$ are the number of particles of the selected component $i$ in the donor and acceptor boxes, respectively, and $V^{d}$ and $V^{a}$ are the volumes of these two boxes. For a mixture of two monomers this move can be used to exchange both components between both boxes. However, for the polymer-solvent system it can only be applied to the solvent particles. The conventional way to move a polymer chain from one box to the other one is by using a CBMC (configurational bias Monte Carlo) algorithm. In our systems this approach is also extremely inefficient, and we will, therefore, introduce an alternative approach;

(3) volume change. The simulation can be performed at a constant total volume (i.e., constant overall density), or at a constant pressure. In the first case, the volume of one of the boxes is increased by $\Delta V$ and the volume of the other box is decreased by the same value. The acceptance probability of this move is

$$
P^{a c}=\min \left(1, \frac{V^{\mathrm{I}}+\Delta V}{V^{\mathrm{I}}} \times \frac{V^{\mathrm{II}}-\Delta V}{V^{\mathrm{II}}} \times \exp (-\beta \Delta U)\right)
$$

(assuming that box I is the box whose volume increases). For a symmetric mixture of monomers $\left(N_{S}=N_{P}\right)$ it is not necessary to include the volume rearrangement in the case of a constant total volume. When the system is simulated at a constant pressure $P^{\text {ext }}$ (which is externally 
imposed on the system), the volume of only one box (say, box I) is changed in one Monte Carlo move (from $V^{\mathrm{I}}$ to $\left.V^{\mathrm{I}}+\Delta V\right)$. In this case the acceptance probability is:

$P^{a c}=\min \left(1,\left(\frac{V^{\mathrm{I}}+\Delta V}{V^{\mathrm{I}}}\right)^{N^{\mathrm{I}}} \times \exp \left(-\beta \Delta U-P^{\operatorname{ext}} \Delta V\right)\right)$,

(4) identity change. In a mixture of two monomers the equilibration can be improved by choosing a random $S$ particle in box I and exchanging it with a randomly chosen $P$ particle in box II (option 1); or, with equal probability, choosing a $P$ particle in box I and exchanging it with a randomly chosen $S$ particle in box II (option 2) [Ref. 17]. This move is then accepted with a probability

$$
P^{a c}=\left\{\begin{array}{l}
\min \left(1, \frac{N_{S}^{\mathrm{I}} N_{P}^{\mathrm{II}}}{\left(N_{S}^{\mathrm{II}}+1\right)\left(N_{P}^{\mathrm{I}}+1\right)} \times \exp (-\beta \Delta U)\right) \\
\text { option 1, } \\
\min \left(1, \frac{N_{P}^{\mathrm{I}} N_{S}^{\mathrm{II}}}{\left(N_{P}^{\mathrm{II}}+1\right)\left(N_{S}^{\mathrm{I}}+1\right)} \times \exp (-\beta \Delta U)\right) \\
\text { option } 2 .
\end{array}\right.
$$

Of course this move cannot be applied directly to a polymer-solvent mixture. For such a system we have developed a new move in which a polymer chain from one box is exchanged with $r$ solvent particles from the other box.

\section{A. identity change move for polymeric systems}

The algorithm for the polymer-solvent Identity change move is as follows:

(1) Choose one of the two boxes, from which the polymer chain is moved to the other one (we assume that box I is chosen);

(2) choose a polymer chain at random from box I, and a solvent particle from box II. The first segment of the polymer chain will be inserted at the position of this solvent particle;

(3) we now move on to the next segment (segment 2, or, more generally, segment $i+1)$. Locate all $K(i)$ " "neighbor particles" of segment $i$, which are defined as the solvent particles that lie within a certain radius $(=$ maximum bond length of the polymer) from segment $i$. We use a value of 1.75 for this radius. (At this point it becomes clear why we introduced the rather steep bonding potential of Eq. (2). For a less steep potential $U^{b}(r)$, the maximum bond length would be longer, giving larger values for $K$. In order to obey the condition of microscopic reversibility, it is necessary to take all possible positions of the new segment into account.)

We now choose one of these $K(i)$ particles to become segment $i+1$. The choice among these particles is made according to the probability distribution $P$ $=\exp \left(-\beta U^{b}\left(r_{i, i+1}\right)\right)$, where $r_{i, i+1}$ is the distance between segment $i$ and the new segment $i+1$. (Of course, it is possible to use a different probability distribution to select the new segment.) Now we calculate the segment factor

$P^{s}(i+1)$

$P^{S}(i+1)=\frac{\exp \left(-\beta U^{b}\left(r_{i, i+1}\right)\right.}{\sum_{j=1}^{K(i)} \exp \left(-\beta U^{b}\left(r_{i j}\right)\right)}$,

where the denominator is a sum over the bonding energies of all "neighbor particles" if they were converted into a chain segment;

(4) this procedure is repeated until $i+1=r$ and $W^{\text {new }}$ is calculated as

$W^{\text {new }}=\prod_{i=1}^{r-1} P^{s}(i+1) ;$

(5) in a completely analogous way $W^{\text {old }}$ is calculated for the old chain conformation in box I, and the energy difference $\Delta U=U^{\text {new }}-U^{\text {old }}$ is calculated for the move;

(6) finally, the move is accepted with a probability

$$
\min \left(1, \frac{N_{S}^{\mathrm{I}} M^{\mathrm{II}}}{\left(N_{S}^{\mathrm{II}}+r\right)\left(M^{\mathrm{I}}+1\right)} \frac{W^{\text {old }}}{W^{\text {new }}} \exp (-\beta \Delta U)\right) .
$$

The procedure outlined above can be compared with a CBMC algorithm for a lattice chain where the lattice sites are formed by the solvent particles. The expression for the acceptance probability of the identity change move is analogous to that of the CBMC move. A formal derivation of this expression is given in the Appendix.

The identity change move for polymers is necessary because it is not possible to exchange a polymer chain directly between both boxes. The approach we have taken bears some likeness with that of the semigrand canonical Gibbs ensemble method proposed by Kofke and Glandt. ${ }^{19}$ In this ensemble one also has a mixture of two different species. Only one of these species is moved from one box to another. In addition, any particle can change its identity without changing its spatial coordinates. For example, Stapleton et al. ${ }^{20}$ used this method to calculate the phase equilibria of fluids of polydisperse particles such as micellar solutions. Müller and Wilding $^{21}$ studied asymmetric binary polymer mixtures (using the bond fluctuation model) in which the chain length of one species is an integer times that of the other one. In our case it would be possible to convert $r$ solvent particles into one polymer chain without exchanging any particles between the boxes, if $N_{S}$ and $N_{P}$ were not kept constant. In combination with the direct exchange of solvent particles, such a semigrand canonical approach would in principle be an alternative for the identity swap move to equilibrate the system.

\section{B. Simulation analysis and mean-field theory}

At the end of the simulation, the results can be analyzed by measuring the average densities of solvent and polymer in both boxes. This method only works when the system is well into the two-phase region. Near the critical point the ideal way to analyze the results would be the histogram method (probability of finding a certain fraction of polymer in one of 


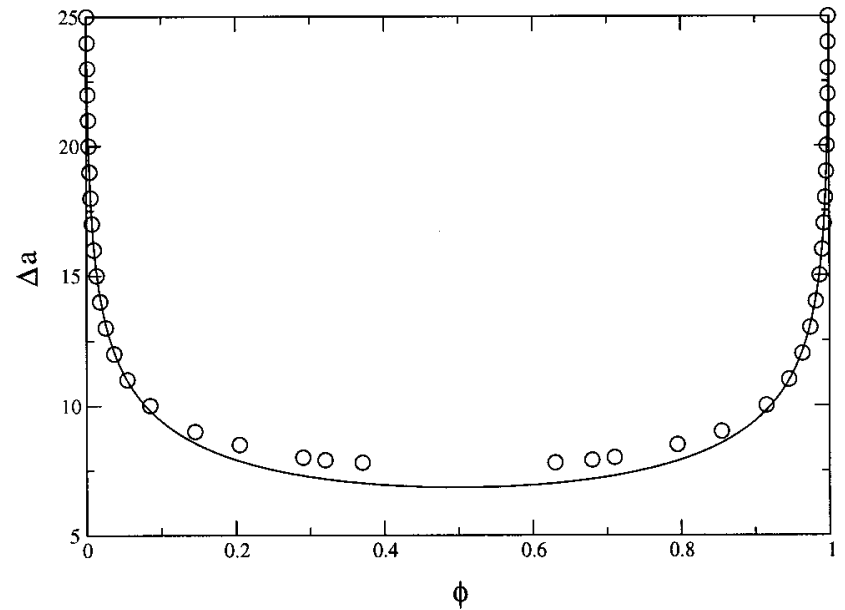

FIG. 1. Phase diagram of a mixture of two different monomers. Parameters: $a_{i i}=25.0, \rho=3.0$. The symbols are simulated data. The curve is the meanfield prediction, as explained in the text.

the boxes plotted versus that fraction). However, in most cases this gives a maximum value of zero for the lower fraction. (That is because of the finite system size and the length of the polymer chains.) Therefore, we calculate the ratios of both species in both boxes at each timestep, and average these ratios at the end of the simulation.

The compositions of the simulated coexisting phases are compared with the predictions of mean-field theory. According to Flory-Huggins theory, in a mixture of polymer (volume fraction $\phi$ ) and solvent (volume fraction $1-\phi$ ) the chemical potentials of the solvent, $\mu_{S}$, and polymer, $\mu_{P}$, can be written as

$$
\begin{aligned}
& \frac{\mu_{S}(\phi)-\mu_{S}(0)}{k T}=\ln (1-\phi)+\left(1-\frac{1}{r}\right) \phi+\chi \phi^{2}, \\
& \frac{\mu_{P}(\phi)-\mu_{P}(1)}{k T}=\ln \phi-(r-1)(1-\phi)+\chi r(1-\phi)^{2},
\end{aligned}
$$

where $\chi$ is the Flory-Huggins interaction parameter. It is now possible to calculate binodal curves from the conditions $\mu_{S}\left(\phi_{\text {low }}\right)=\mu_{S}\left(\phi_{\text {high }}\right)$ and $\mu_{P}\left(\phi_{\text {low }}\right)=\mu_{P}\left(\phi_{\text {high }}\right)$, where $\phi_{\text {high }}$ and $\phi_{\text {low }}$ are the polymer volume fractions in the two coexisting phases for a given value of $\chi$. For $r=1$ one gets the following expression for the relationship between $\phi$ and $\chi$ :

$$
\chi=\frac{\ln \left(\frac{1-\phi}{\phi}\right)}{1-2 \phi} .
$$

\section{RESULTS AND DISCUSSION}

\section{A. Monomeric mixtures}

Figure 1 shows simulated data of the phase behavior of a system with 1000 particles $\left(N_{S}=N_{P}=500\right)$ at a total density $\rho=3.0$, for $a_{i i}=25.0$. The value of $\Delta a$ has been plotted as a function of the particle molar fraction $\phi$. The symbols in

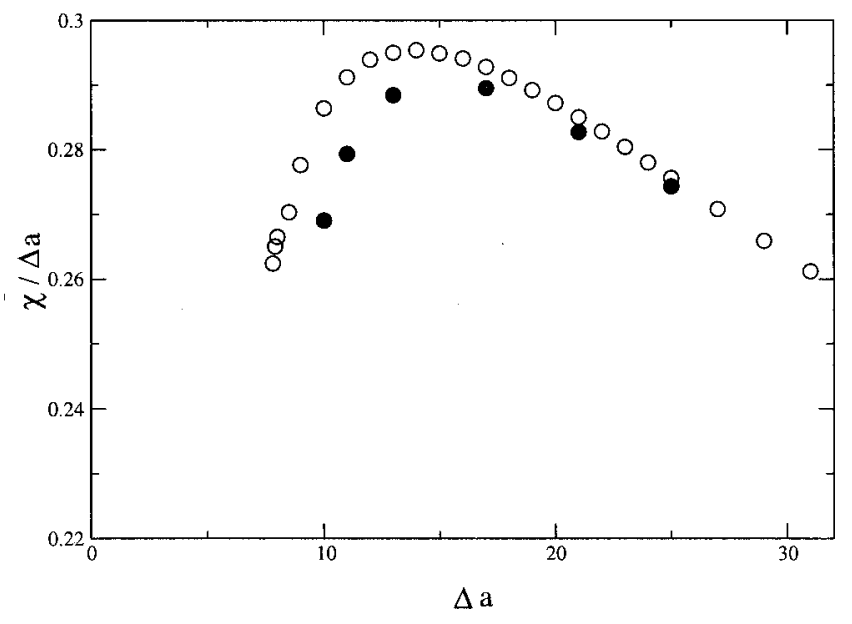

FIG. 2. Ratio of the effective $\chi$-parameter and $\Delta a$ for constant volume simulations (open symbols) and constant pressure simulations (filled symbols) $(\rho=3.0)$.

Fig. 1 are results from the Gibbs ensemble simulations, whereas the curve is a mean-field fit which will be explained below.

Equation (8) has been used to calculate the effective value of $\chi$ as a function of $\Delta a$ for the phase separation data of Fig. 1 (at constant volume). In Fig. 2 the ratio $\chi / \Delta a$ has been plotted against $\Delta a$ (open symbols). The filled symbols in Fig. 2 are derived from constant pressure simulations, which will be discussed later.

It can be seen from Fig. 2 that $\chi$ is not a linear function of $\Delta a$. However, if, as a first-order approximation, we would assume that $\chi$ is proportional to $\Delta a$, we get the following relationship based on a fit of the simulated data in the range $10.0 \leqslant \Delta a \leqslant 20.0$ (i.e., not too near the critical point):

$$
\chi=(0.292 \pm 0.003) \Delta a .
$$

This corresponds well with Ref. 5, where it is reported that $\chi=(0.286 \pm 0.002) \Delta a$. The error given in Eq. (9) represents the systematic deviation of $\chi / \Delta a$ with $\Delta a$. The curve in Fig. 1 was drawn by combining Eqs. (9) and (8). Except near the critical point, this " linear' mean-field approximation gives a reasonably good prediction of the simulated data.

If we take the mean-field expression for the phase equilibrium as definition of the interaction parameter $\chi$, then $\chi$ is given by the following difference in free energy:

$$
\chi_{i j}=\frac{1}{2 k T}\left(\frac{\Delta \mu_{i i}-\Delta \mu_{i j}}{V_{i}}+\frac{\Delta \mu_{j j}-\Delta \mu_{j i}}{V_{j}}\right),
$$

where $\Delta \mu_{i j}$ is the excess chemical potential (with respect to an arbitrary reference state) of species $i$ in an environment $j$, and $V_{i}$ is the molecular volume of $i$. We consider a system of $i$-particles only, and we compare this system with another system in which one $i$-particle has been replaced by a $j$-particle. If we only consider the interaction energy $U_{i j}$ of the $j$-particle with all surrounding $i$-particles, we can make a first approximation of $\chi$ as

$$
\chi=U_{i j}(\Delta a)-U_{i j}(\Delta a=0) .
$$




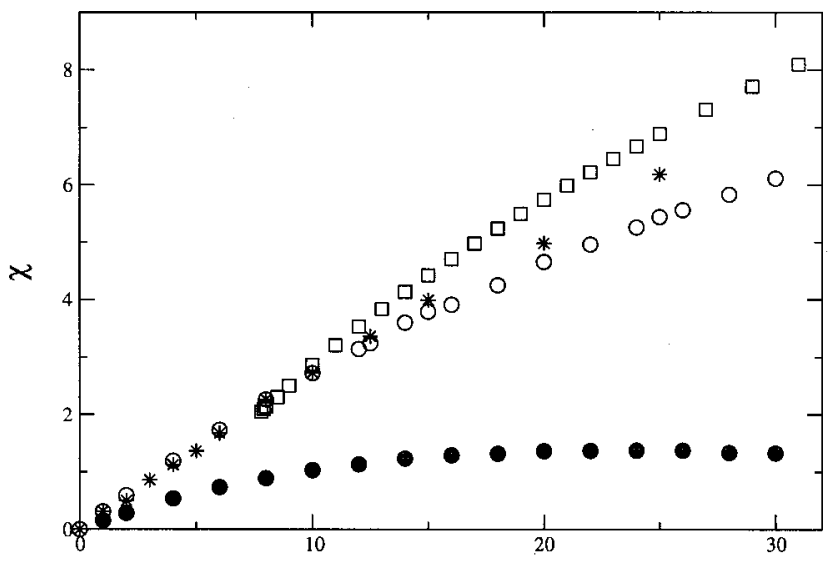

$\Delta \mathrm{a}$

FIG. 3. Relationship between different expressions for the $\chi$-parameter and the excess repulsion $\Delta a$. In all cases $\rho=3.0$. Open circles: Eq. (12) for $a_{i i}=25.0$. Stars: Eq. (12) for $a_{i i}=75.0$. Filled circles: Eq. (11) for $a_{i i}$ $=25.0$. Squares: Eq. (8) for $a_{i i}=25.0$.

This expression is exact for a lattice model (without vacancies), in which $\chi$ is simply defined as $\chi_{i j}=z / 2\left(2 w_{i j}-w_{i i}\right.$ $-w_{j j}$ ), where $z$ is the lattice coordination number and $w_{i j}$ is the interaction energy between two particles $i$ and $j$ on neighboring sites. In general, a more correct expression for $\chi$ considers all interactions rather than only the $i-j$ interactions. This leads to the following approximation for $\chi$ :

$$
\chi=U^{\text {total }}(\Delta a)-U^{\text {total }}(\Delta a=0) .
$$

Equation (12) takes multibody correlations into account but still neglects the entropic contributions to the free energy.

In Fig. 3 results of Eqs. (11) (filled circles) and (12) (open circles) are shown for a system with 375 particles at a density $\rho=3.0$, using a repulsion parameter $a_{i i}=25.0$. The stars are the results of Eq. (12) for a system with $a_{i i}$ $=75.0$. The squares give the $\chi$ values that are directly calculated from the phase equilibrium using Eq. (8). The system phase behavior hardly depends on the value of $a_{i i}$, but only on $\Delta a$. This explains the very close agreement of the results of Eq. (12) for $a_{i i}=25.0$ and $a_{i i}=75.0$ (for $\Delta a$ up to 10). It is clear from Fig. 3 that Eq. (11) does not give a good prediction of $\chi$. When all interactions are taken into account [Eq. (12)] one gets a far better agreement with the values for $\chi$ that are found directly from the phase diagram using Eq. (8) than when only the interactions between particle $j$ and its neighbors are taken into consideration [Eq. (11)]. But for large values of $\Delta a(\Delta a>10)$, Eq. (12) does still underestimate $\chi$.

The difference between Eqs. (11) and (12) is due to the rearrangement of $i$-particles around the $j$-particle (three-body correlations). The (excess) repulsion by the $j$-particle means that the $i$-particles will want to move away from this particle. Consequently, the average distance between $i$-particles will decrease and the potential energy due the interactions between $i$-particles will also increase. At moderately large $\Delta a$, three-body correlations are clearly the most important factor determining $\chi$.

Figure 4 shows the radial distribution function $g_{i j}(r)$ of

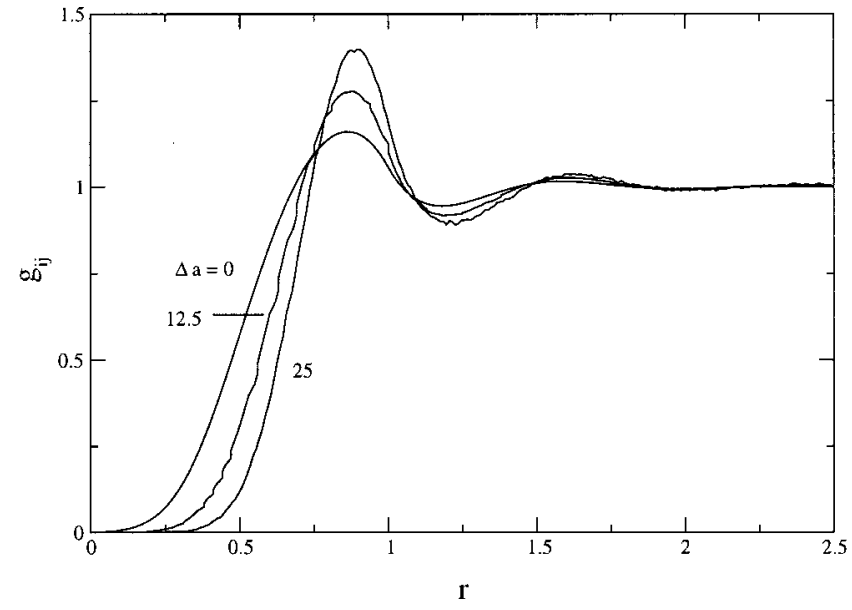

FIG. 4. Radial distribution functions $g_{i j}(r)$ of the $i$-particles with respect to the $j$-particle for different excess repulsions as indicated in the graph.

$i$-particles around the $j$-particle for three different values of the repulsion parameter: $\Delta a=0,12.5,25.0\left(a_{i i}=25.0\right.$ and $\rho=3.0)$. On average, the $i$-particles do indeed move farther away from the $j$-particle as $\Delta a$ increases. This implies that the $i$-particles become more strongly ordered as the repulsion increases, and therefore the entropy of the system increases.

This free energy difference (between a system with $N$ $i$-particles and one with $N-1 i$-particles and one $j$-particle) can be computed using the Kirkwood coupling parameter method [Ref. 18]. We introduce a coupling parameter $\lambda$ which increases linearly from zero to unity as $\Delta a$ increases from zero to its maximum value. We then calculate the free energy difference as

$$
\Delta F(\Delta a)=\int_{0}^{1} \mathrm{~d} \lambda\left\langle\frac{\partial U(\lambda)}{\partial \lambda}\right\rangle_{\lambda},
$$

where $U(\lambda)$ is the system energy for $a_{i j}=a_{i i}+\lambda \Delta a$. This energy is the sum of two terms, $U_{i i}$ and $U_{i j}$, where the first term accounts for all $i-i$ interactions and the second one accounts for all $j-j$ interactions. The partial derivative $\partial U_{i i} / \partial \lambda$ equals zero. The partial derivative of $U$ with respect to $\lambda$ is calculated as

$$
\left\langle\frac{\partial U(\lambda)}{\partial \lambda}\right\rangle_{\lambda}=\left\langle\frac{\partial U_{i j}(\lambda)}{\partial \lambda}\right\rangle_{\lambda}=U_{i j}(\lambda) \times \frac{\Delta a}{a_{i i}+\lambda \Delta a} .
$$

In Fig. 5 both $\Delta U$ and $\Delta F$ are plotted as a function of $\Delta a$ for the system $\left(\rho=3.0, a_{i i}=25.0\right)$. These values are compared with $\chi$ as calculated using Eq. (8). As $\Delta a$ increases (beyond $\sim 15$ ), $\Delta U$ clearly underestimates $\chi$, but the free energy difference $\Delta F$ gives a very good estimate of $\chi$. The line given by Eq. (9) is also drawn in Fig. 5. Although the effective $\chi$ values do not lie exactly on this line, this simple expression can be used as a reasonable first-order approximation for the relationship between $\chi$ and $\Delta a$. For small values of $\Delta a$, where the monomers do not phase separate, this linear expression still agrees well with the values of $\Delta F$. Polymers will phase separate at these smaller $\Delta a$ values, so we expect to be able to use Eq. (9) to calculate the relevant $\chi$ parameters for polymer-solvent systems. 


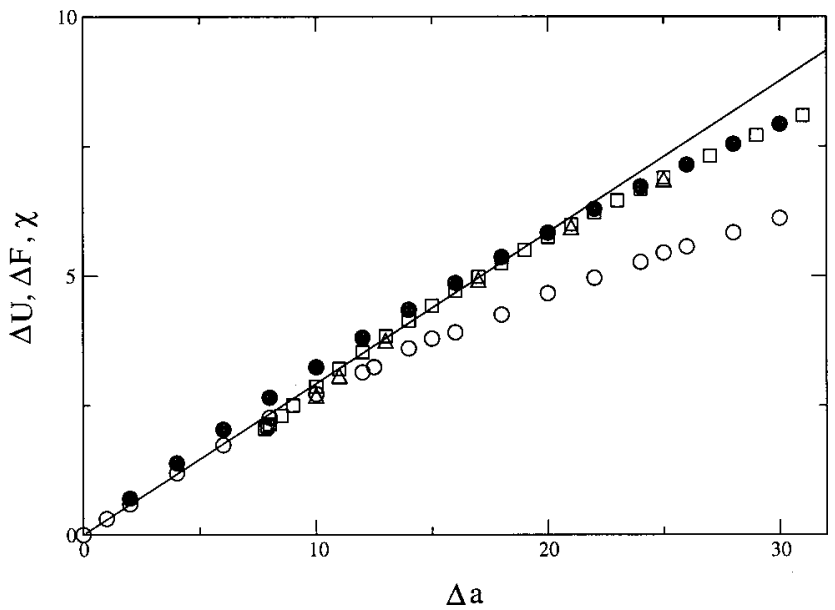

FIG. 5. Energy difference (open circles) and free energy difference (filled circles) between a system with $N-1 i$-particles and $1 j$-particle and a system with $N i$-particles. The squares (constant volume simulations) and triangles (constant pressure simulations) give $\chi$ according to Eq. (8). The line shows the linear relationship between $\chi$ and $\Delta a$ of Eq. (9).

One factor that we have ignored up to now is the effect of pressure. The simulation results shown up to now were all at constant volume. These simulations have been repeated at constant pressure, using the pressure of a system of particles with a density $\rho=3.0$ and a repulsion parameter $a=25.0$ for all interactions (which is $P=23.83$ in simulation units). The results from the constant pressure simulations have been included in Figs. 2 and 5. Figure 2 shows that for any value of $\Delta a$, the effective $\chi$ value is slightly smaller in a constant pressure simulation than in a constant volume simulation. The difference between both simulations is a few percent. Figure 5 shows that the free energy difference $\Delta F$ gives a good prediction for $\chi$ both in the constant volume and in the constant pressure simulations.

\section{B. Polymer-solvent mixtures}

In Fig. 6 phase diagrams are shown for a chain length $r=20$. In all cases the particle density $\rho=3.0$ (all simulations were conducted at constant total volume). All open symbols refer to simulations with a repulsion parameter between like particles (solvent-solvent and polymer-polymer) $a_{i i}=25.0$. The total number of particles in these simulations $\left(N=N_{S}+M r\right)$ varies from 1000 to 4000 . Different system sizes were used to check for finite system size effects. The filled symbols refer to simulations with $a_{i i}=75.0$. In this case $N=2000$ only. The curves in Fig. 6 are mean-field predictions. They were calculated using Eqs. (6), (7), and (9).

Figure 6 demonstrates the potential of the identity swap move to equilibrate a polymer-solvent mixture. For the systems shown here, the solvent insertion acceptance probability is $\sim 0.5$ for $a_{i i}=25$, and two orders of magnitude smaller for $a_{i i}=75$. Similar values would be found for polymer segments, and, although these values are not extremely small, they do lead to very small values for the acceptance probability of long chains using a CBMC algorithm. In contrast, the identity change move has an acceptance probability which is as large as 0.24 for $a_{i i}=25$ and $\Delta a=3.5$. As $\Delta a$ increases, this probability decreases, so that in practice there
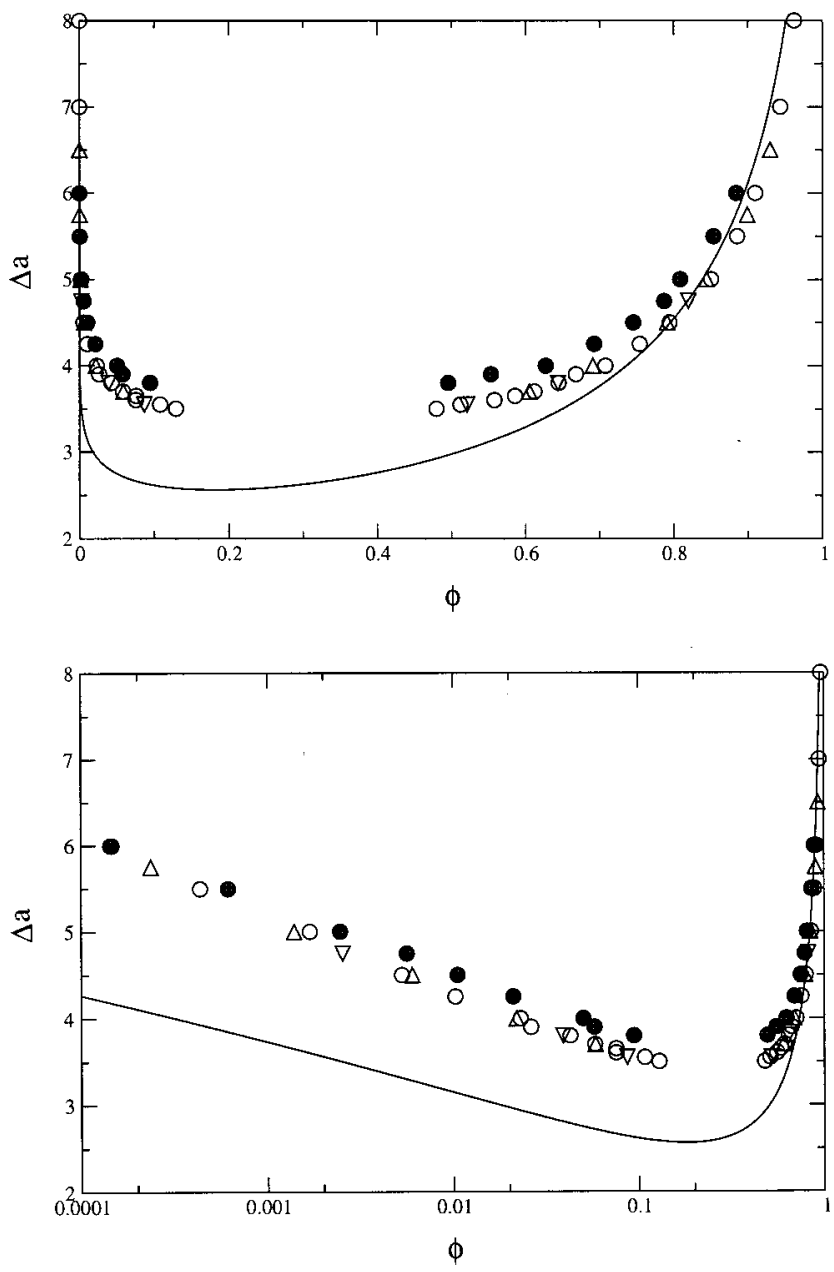

FIG. 6. Phase diagrams of polymer-solvent mixtures for $r=20 ; N=1000$ (triangles up), $N=2000$ (circles), and $N=4000$ (triangles down). Two different values were used for $a_{i i}: a_{i i}=25.0$ (filled symbols) and 75.0 (open symbols). In both cases the density is $\rho=3.0$. The full curves show the Flory-Huggins binodals, based on the relationship between $\chi$ and $\Delta a$ given by Eq. (9).

is always an upper limit on the value of $\Delta a$ for which it is possible to simulate the binodal curve. For example, for $\Delta a=5.0$ we have an acceptance probability of 0.009 . For longer chain lengths these probabilities are smaller and decrease more quickly with increasing $\Delta a$. No finite size effects show up when one compares the results of simulations using different system sizes.

In Fig. 6 there is a small but systematic difference between the results for $a_{i i}=25$ and $a_{i i}=75$. The data for $a_{i i}$ $=75$ are shifted to slightly higher values of $\Delta a$. Although better statistics are required to provide a very precise determination of the critical point, it is clear that the critical point occurs for a higher value of $\Delta a$ than predicted by the FloryHuggins curve (a difference of $\sim 30 \%$ ). For the monomeric system the difference between the value of $\Delta a$ at the critical point predicted by the mean-field theory and the value found in the simulations was $\sim 13 \%$. Although this difference seems smaller than that for the polymer solutions, one must bear in mind that the absolute value of $\Delta a$ at the critical point is more than twice as large in the monomer system compared to the polymer system. Moving away from the 


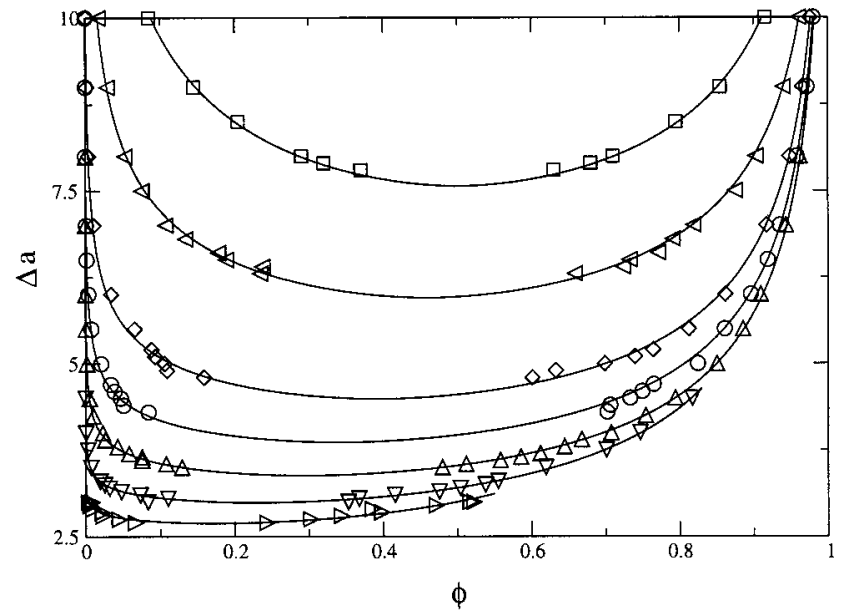

FIG. 7. Phase diagrams of polymer-solvent mixtures for $r=1$ (squares), 2 (triangles left), 5 (diamonds), 10 (circles), 20 (triangles up), 50 (triangles down), and 150 (triangles, right). The symbols are simulation data. The curves show the fit of Eq. (16) using the parameter values given in Table I.

critical point, the polymer concentration in the concentrated polymer phase is seen to be fairly well predicted by the Flory-Huggins curve. However, in the low-polymer concentration phase, the polymer concentrations are far higher than predicted by Flory-Huggins theory [see Fig. 6(b)]. For example, for $a_{i i}=25$ the simulations give a polymer fraction of $2 \times 10^{-2}$, whereas the mean-field value is $5 \times 10^{-5}$. This is not surprising, as the mean-field approximation greatly underestimates the stability of the polymer chains in the dilute regime.

Binodal curves were simulated for a series of chain lengths, ranging from $r=1$ up to 150 . In all cases we used $a_{i i}=25.0$. The symbols in Fig. 7 show the results of these simulation runs. In order to arrive at closed expressions we derived equations that give a good fit of the simulated data. These curves are also shown in Fig. 7. Below, we explain how they were calculated.

For $r=1$ the binodal curve $\Delta a(x)$ is a symmetric function of $x=\ln ((1-\phi) / \phi)$, and a power-law in this coordinate gives a very accurate description of the simulation data. We find

$$
\Delta a=c_{1}\left|\ln \left(\frac{1-\phi}{\phi}\right)\right|^{c_{2}}+c_{3},
$$

with $\quad c_{1}=0.5159 \pm 0.0038, \quad c_{2}=1.8259 \pm 0.0035, \quad$ and $c_{3}$ $=7.569 \pm 0.014$. (Surprisingly, the power $c_{2}$ is less than 2.) The correlation coefficient of this fit is $R^{2}=0.999973$, and Fig. 7 also shows that the fit is excellent down to the critical point.

For longer chain lengths $(r>1)$ we introduce a variable $n$ which depends on the polymer chain length, and we now fit the binodal curve to the function $\Delta a(x)$, where $x$ $=n \ln (1-\phi)-\ln (\phi)$. This gives a four-parameter fit; However, the fitting parameters appear not to be completely independent, and the errors become very large. We therefore fixed the power $c_{2}$ at the value given above, resulting in the following three-parameter fit function:
TABLE I. Fit parameters for Eq. (16) to describe the simulated polymer binodal data. The numbers in brackets are the estimated errors in the last digits. The column marked $\phi_{c}$ gives the volume fraction at the critical point calculated from Eq. (17). $R^{2}$ is the correlation coefficient for the fit. In addition to the chain lengths shown in Fig. 7, three more chain lengths $(r=14,32$, and 85$)$ were simulated.

\begin{tabular}{rlllll}
\hline \hline$r$ & \multicolumn{1}{c}{$c$} & \multicolumn{1}{c}{$n$} & \multicolumn{1}{c}{$\Delta a_{c}$} & $\phi_{c}$ & \multicolumn{1}{c}{$R^{2}$} \\
\hline 1 & $0.5157(5)$ & 1.0000 & $7.569(9)$ & 0.5000 & 0.999973 \\
2 & $0.308(6)$ & $1.27(1)$ & $5.95(3)$ & 0.4589 & 0.995 \\
5 & $0.154(2)$ & $1.89(2)$ & $4.49(3)$ & 0.3913 & 0.997 \\
10 & $0.088(1)$ & $2.60(2)$ & $3.86(3)$ & 0.3398 & 0.996 \\
14 & $0.073(1)$ & $2.90(3)$ & $3.59(1)$ & 0.3228 & 0.996 \\
20 & $0.053(7)$ & $3.51(3)$ & $3.38(1)$ & 0.2943 & 0.997 \\
32 & $0.039(2)$ & $4.33(6)$ & $3.15(2)$ & 0.2645 & 0.97 \\
50 & $0.0262(6)$ & $5.56(8)$ & $2.99(1)$ & 0.2315 & 0.992 \\
85 & $0.020(1)$ & $7.0(2)$ & $2.84(2)$ & 0.2035 & 0.95 \\
150 & $0.14(1)$ & $8.9(4)$ & $2.69(1)$ & 0.1768 & 0.88 \\
\hline \hline
\end{tabular}

$$
\Delta a=c\left|\ln \left(\frac{(1-\phi)^{n}}{\phi}\right)\right|^{1.826}+\Delta a_{c} .
$$

This equation was used to draw the curves shown in Fig. 7. The values of the fitting parameters and the correlation coefficients for different chain lengths are given in Table I. Once we have the functional fit to the binodal, the volume fraction at the critical point follows from the minimum in $\Delta a(\phi)$, which occurs at

$$
(1-\phi)^{n}=\phi,
$$

and which can be solved numerically.

To arrive at a completely closed expression for the binodal curves, we will derive functions describing the dependence of the fit parameters in Table I on the chain length $r$. We start by considering the relationship between the critical volume fraction $\phi_{c}$ and the chain length $r$. The mean-field expression for $\phi_{c}$ is

$$
\phi_{c}=\frac{1}{1+r^{1 / 2}} .
$$

By generalizing this expression and imposing the exact condition that $\phi_{c}=1 / 2$ at $r=1$, we can fit $\phi_{c}$ to the function

$$
\phi_{c}=\frac{b}{2 b-1+r^{x}} .
$$

We then find that $b=1.53 \pm 0.09$ and $x=0.38 \pm 0.01$. Combining Eqs. (18) and (17), we get a closed functional form for $n$

$$
n=\frac{\ln \frac{b}{2 b-1+r^{x}}}{\ln \frac{b-1+r^{x}}{2 b-1+r^{x}} .}
$$

When we separately fit the data for $n$ to this expression (as $n$ was the actual parameter in our fits, and not $\phi_{c}$ ), we find 
$b=1.36 \pm 0.09$ and $x=0.36 \pm 0.01$. It should be noted that $n$ has also been fitted by a power-law of $r\left(n \sim r^{0.435}\right)$, but the worse quality of that fit rules out a simple power-law for $n$ on the basis of our data, in favor of Eq. (20).

The mean exponent $x=0.37 \pm 0.01$ that we find from the simulated data can be compared with several experimental and simulation studies. Dobashi et al. ${ }^{22}$ measured coexistence curves for polystyrene-methylcyclohexane mixtures which give a value $x=0.39$. Shinozaki et al. ${ }^{23}$ reported a value of $x=0.46$ for polystyrene-propylbenzene mixtures. Simulations of lattice polymers have yielded values for this exponent in the range $0.27-0.38$. Mackie $e t$ al. ${ }^{24}$ reported an exponent of $x=0.32$, although this value increases to 0.35 if they only consider their longest chain length simulations. Wilding et al. ${ }^{25}$ found $x=0.37$ and Yan et al. ${ }^{26}$ reported that $x=0.27$. Finally, Panagiotopoulos et al. ${ }^{27}$ found a value $x$ $=0.38$. Our exponent clearly falls within the range of values found in the lattice simulations. The Flory-Huggins exponent $(x=0.5)$ very clearly falls outside this range of values.

We still need to consider the parameters $c$ and $\Delta a_{c}$ in Eq. (16). The former parameter is well described by a powerlaw of $r$. Forcing this expression through its $r=1$ value, which is our most accurate result, we obtain

$$
c=0.5159 r^{-0.751(4)} .
$$

Finally, for $\Delta a_{c}$ we try a functional form that is similar to that of the mean-field expression for the critical FloryHuggins parameter $\chi_{c}$

$$
\chi_{c}=\frac{1}{2}\left(1+\frac{1}{r^{1 / 2}}\right)^{2},
$$

but we replace the powers $1 / 2$ and 2 by free parameters. Forcing this fit through the $r=1$ result $\left(\Delta a_{c}=7.569\right)$, we obtain

$$
\Delta a_{c}=7.569\left(\frac{1+r^{-0.446(9)}}{2}\right)^{1.75(2)} .
$$

Equation (23) predicts that for $r \rightarrow \infty$ we will find an excess critical repulsion $\Delta a_{c}=2.25$, which is a $31 \%$ larger value than predicted by mean-field theory. The fact that for infinite chain length we do not recover the scaling behavior predicted by Flory-Huggins theory indicates that there is a qualitative difference between a polymer-solvent mixture and a binary polymer blend, which was simulated by Escobedo and de $\mathrm{Pablo}^{28}$ and which only deviates from the simple scaling law $T_{c} \sim N$ for finite $N$.

The closed expression for the binodal is finally found by combining the previous results, which lead to

$$
\begin{aligned}
\Delta a= & 0.516 r^{-0.751}|n \ln (1-\phi)-\ln (\phi)|^{1.826} \\
& +2.25\left(1+r^{-0.44}\right)^{1.75} .
\end{aligned}
$$

Equation (24) enables us to compare the simulations directly with experimental binodal curves. We have used the phase equilibrium data of the polystyrenemethylcyclohexane system given in Ref. 22 for this purpose.

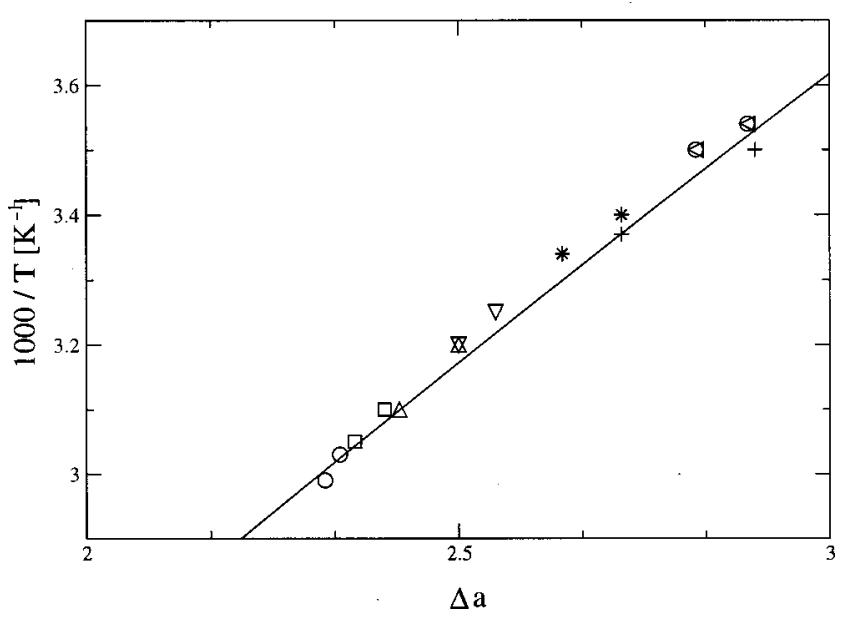

FIG. 8. Inverse temperature as a function of $\Delta a$. Each pair of symbols indicates the end points of the line segment for one experimental polymer sample. The curve is an overall (power-law) fit.

We analyzed the experimental data for eight different molecular weights $\left(10^{-4} M_{w}=1.02,1.73,2.02,3.49,4.64,10.9\right.$, $18.1,71.9)$. Defining a parameter $\beta=1000 / T$, where $T$ is the coexistence temperature, we mapped the experimental data points $\beta\left(\phi, M_{w}\right)$ onto $\Delta a(\phi, r)$. For each molecular weight we fitted the experimental binodal to the function

$$
\beta=\lambda \Delta a+\mu,
$$

where $\Delta a$ is the function given in Eq. (24), and where $\lambda, \mu$, and $r^{\prime}$ are fitting parameters. For each molecular weight this gives a linear relation between $\beta$ and $\Delta a$, which is valid over a small range of temperatures. These results are collected in Fig. 8. Different symbols indicate the end points of the line segments for one particular binodal, calculated for the lowest and highest temperature available for that particular polymer sample. The curve is an overall fit to these points. Theoretically all line segments should fall on this mean curve. It is obvious that only the result for the highest molecular weight (the lowest $\Delta a$ ) has a slope that differs considerably from the mean; all other experiments are reasonably consistent. The curve now represents the mapping between temperature and excess repulsion; it is given by

$$
\beta=(1.64 \pm 0.03) \Delta a^{0.72 \pm 0.02} .
$$

Having determined the relationship between the temperature and the excess repulsion parameter, the chain length $r$ remains as the only free parameter to fit Eq. (24) to the experimental data. The result of this fit is shown in Fig. 9. A good agreement is found between the experimental data and the simulated expression for the binodal curves. Figure 10 shows the values of $r$ that were found in the fitting procedure. The chain length $r$ should scale linearly with the molecular weight of the polymer samples. We find a relationship $r \propto M_{w}^{1.13}$, which is reasonably consistent with the actual molecular weight. The sample with the largest molecular weight again does not agree very well with the other data points. 


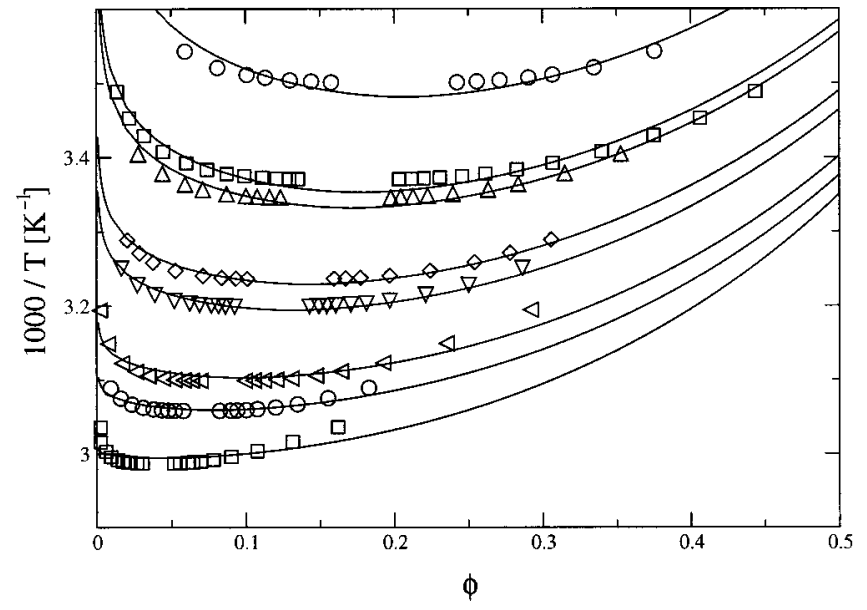

FIG. 9. Comparison of the fit function given by Eq. (24) with experimental phase coexistence data for the polystyrene-methylcyclohexane system taken from Ref. 22. The polymer molecular weights are given in the text. All curves were fitted using the same mapping between $T$ and $\Delta a$.

\section{CONCLUDING REMARKS}

In this paper we have presented Gibbs ensemble Monte Carlo simulations to investigate the phase behavior of systems with soft potentials such as those that are used in the DPD simulation technique. As a first-order approximation it is possible to interpret the behavior of such systems in terms of a Flory-Huggins $\chi$ parameter that is proportional to the repulsion parameter difference $\Delta a$ [Eq. (9)]. This approach was first suggested in Ref. 5 as a way to bridge the gap between mesoscopic DPD simulations and real systems with experimental parameters. We have shown in this paper that the above definition of $\chi$ agrees reasonably well with the free energy difference between a monomer surrounded by solvent particles, and a solvent particle surrounded by solvent particles. However, near the critical point the mean-field description of a monomermonomer mixture shows a clear discrepancy with simulated phase coexistence equilibria.

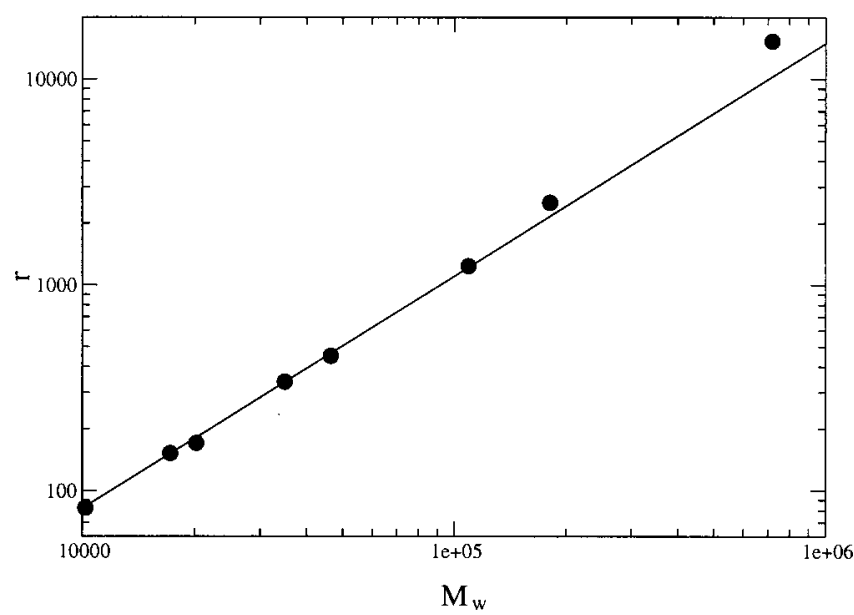

FIG. 10. Polymer chain length $r$ in the fitting procedure as a function of the experimental molecular weight. The line has a slope of 1.13.
In addition to monomer-monomer mixtures, we have simulated the phase behavior of polymer-solvent mixtures. In order to equilibrate such systems it was necessary to develop a new "identity change" Monte Carlo move. Our simulation method can also be used for models with a different type of pair potential, as long as the monomers and solvent particles are of the same, or at least similar, size. We demonstrated the potential of this approach for polymer chains up to 150 segments, although this chain length is certainly not an upper limit. A straightforward application of Flory-Huggins theory gives only a rather poor description of the simulation results. The critical point is underestimated (by $\sim 30 \%$ ) and the polymer volume fraction in the low-polymer phase is underestimated by several orders of magnitude. One reason for these discrepancies could be that a Flory-Huggins segment does not correspond exactly to one monomer in our model. However, this can certainly not explain fully the differences between theory and simulation. The main reason for the failure of Flory-Huggins theory is that the mean-field approximation does not give an appropriate description of the system. Of course, one does not expect mean-field to be correct for the very low polymer concentrations that occur in the simulations, where the polymer chains become collapsed globules.

We have fitted a master equation that describes the simulated data for all chain lengths. Such a fit function is a useful tool when one wants to compare the simulations with experimental data. We illustrated how such a comparison can be made with solubility data for polystyrene in methylcyclohexane. The experimental binodal curves can be fitted very well for each individual molecular weight, although the scaling of the the chain length with the experimental molecular weight is not perfect.

Polymer phase coexistence curves have been published for significantly longer chain lengths (up to 1000) using lattice models, ${ }^{24,25,27}$ that were simulated using the Gibbs ensemble and grand-canonical ensemble methodologies. In a lattice model a solvent molecule can simply be represented as an empty lattice site. That makes it a lot easier to equilibrate the system by growing chains on the empty sites. Vapor-liquid equilibria of chain molecules have also been simulated using continuous-space models (up to 100 segments chain length $\left.{ }^{29}\right)$. It is of course possible to regard the vacuum as a solvent, and then one can use the conventional CBMC algorithm to grow the chains. To our best knowledge, this paper presents the first results of simulations of the phase behavior of polymer-solvent mixtures that take explicit account of the solvent molecules.

\section{ACKNOWLEDGMENT}

C.M.W. gratefully acknowledges Unilever Research for financial support. 


\section{APPENDIX}

For a Gibbs ensemble with $N_{S}$ solvent particles and $M$ polymer chains we can write the following expression for the canonical partition function:

$$
\begin{aligned}
Q_{\mathrm{NPT}}^{\mathrm{Gibbs}}= & \frac{1}{N_{S} ! \Lambda_{S}^{3 N_{S}} V} \frac{1}{M ! \Lambda_{P}^{3 N_{P}} V_{N_{S}^{\mathrm{I}}=0}^{N_{S}}} \frac{N_{S} !}{N_{S}^{\mathrm{I}} ! N_{S}^{\mathrm{II} !}} \\
& \times \sum_{M^{\mathrm{I}}=0}^{M} \frac{M !}{M^{\mathrm{I} !} ! M^{\mathrm{II}} !} \int_{0}^{\infty} d V^{\mathrm{I}} \\
& \times \exp \left(-\beta P V^{\mathrm{I}}\right) \int_{0}^{\infty} d V^{\mathrm{II}} \exp \left(-\beta P V^{\mathrm{II}}\right) \\
& \times \int d\left(\mathbf{r}_{S}^{\mathrm{I}}\right)^{N_{S}^{\mathrm{I}}} \int d\left(\mathbf{r}_{P}^{\mathrm{I}}\right)^{N_{P}^{\mathrm{I}}} \exp \left(-\beta U^{\mathrm{I}}\left(\mathbf{r}^{\mathrm{I}}\right)\right) \\
& \times \int d\left(\mathbf{r}_{S}^{\mathrm{II}}\right)^{N_{S}^{\mathrm{II}}} \int d\left(\mathbf{r}_{P}^{\mathrm{II}}\right)^{N_{P}^{\mathrm{II}}} \exp \left(-\beta U^{\mathrm{II}}\left(\mathbf{r}^{\mathrm{II}}\right)\right),
\end{aligned}
$$

where $\mathbf{r}_{i}^{j}$ represents the positions of all particles of type $i$ $\left(i=P\right.$ or $S$ ) in subsystem $j$ and $U^{j}$ is the total energy of subsystem $j$, which is a function of the coordinates of all particles in that subsystem (and consists of both the bonded and nonbonded interactions). This expression is similar to the one given by Smit and Frenkel ${ }^{30}$ and Green et al. ${ }^{31}$ but the segments in the polymer chains are not indistinguishable (as is the case for detached monomers). The kinetic energy of the system is accounted for by the de Broglie wavelengths $\Lambda_{S}$ and $\Lambda_{P}$ of the solvent particles and polymer segments. Following the procedure outlines in Refs. 30 and 31 we introduce a pseudo-Boltzmann factor $P^{\text {Gibbs }}$, which represents the distribution probability of the ensemble average

$$
\begin{aligned}
\ln P^{\mathrm{Gibbs}}= & \ln \left(\frac{N_{S} !}{N_{S}^{\mathrm{I}} ! N_{S}^{\mathrm{II}} !}\right)+\ln \left(\frac{M !}{M^{\mathrm{I}} ! M^{\mathrm{II}} !}\right)+N^{\mathrm{I}} \ln V^{\mathrm{I}}+N^{\mathrm{II}} \ln V^{\mathrm{II}} \\
& -\beta P V^{\mathrm{I}}-\beta P V^{\mathrm{II}}-\beta U^{\mathrm{I}}-\beta U^{\mathrm{II}} .
\end{aligned}
$$

We consider a move in which the system undergoes a transition from configuration $\alpha\left(N_{S}^{a}, M^{a}, N_{S}^{b}, M^{b}\right)$ to configuration $\beta\left(N_{S}^{a}-r, M^{a}+1, N_{S}^{b}+r, M^{b}-1\right)$ by transferring a polymer chain from box II to box I, and $r$ solvent particles from box I to box II. The probability of this transition is proportional to

$$
\begin{aligned}
P_{\alpha \beta}= & N_{S}^{\mathrm{I}} ! M^{\mathrm{I}} ! N_{S}^{\mathrm{II} !} ! M^{\mathrm{II} !} ! \times P^{\mathrm{move}} \times P_{\alpha}^{\mathrm{pol}} \\
& \times P_{\alpha}^{\mathrm{insertion}} \times P_{\alpha}^{\mathrm{Gibbs}} \times P_{\alpha \beta}^{a c},
\end{aligned}
$$

where $P^{\text {move }}=1 / 2$ is the probability that a polymer chain is moved from box II to box I (rather than vice versa), $P_{\alpha \beta}^{\text {ac }}$ is the acceptance criterion for this move, $P_{\alpha}^{\mathrm{pol}}=1 / M^{\mathrm{II}}$ is the probability of choosing one specific polymer chain from box II, $P_{\alpha}^{\text {insertion }}$ is the probability to select a given conformation for the chain that is inserted into box I

$$
P_{\alpha}^{\mathrm{insertion}}=\frac{1}{N_{S}^{\mathrm{I}}} \prod_{i=2}^{r} P_{\alpha}^{s}(i)
$$

and

$$
\begin{aligned}
P_{\alpha}^{\mathrm{Gibbs}}= & \frac{N_{S} !}{N_{S}^{\mathrm{I}} ! N_{S}^{\mathrm{II} !}} \times \frac{M !}{M^{\mathrm{I}} ! M^{\mathrm{II}} !} \times \exp \left(N^{\mathrm{I}} \ln V^{\mathrm{I}}+N^{\mathrm{II}} \ln V^{\mathrm{II}}\right. \\
& \left.-\beta P V^{\mathrm{I}}-\beta P V^{\mathrm{II}}-\beta U_{\alpha}^{\mathrm{I}}-\beta U_{\alpha}^{\mathrm{II}}\right) .
\end{aligned}
$$

For the reverse move we can write

$$
\begin{aligned}
P_{\beta \alpha}= & \left(N_{S}^{\mathrm{I}}-r\right) !\left(M^{\mathrm{I}}+1\right) !\left(N_{S}^{\mathrm{II}}+r\right) !\left(M^{\mathrm{II}}-1\right) ! \\
& \times P^{\mathrm{move}} \times P_{\beta}^{\mathrm{pol}} \times P_{\beta}^{\mathrm{insertion}} \times P_{\beta}^{\text {Gibbs }} \times P_{\beta \alpha}^{\mathrm{ac}},
\end{aligned}
$$

where $P_{\beta}^{\mathrm{pol}}=1 /\left(M^{\mathrm{I}}+1\right)$, the probability to select the right conformation for the chain is

$$
P_{\beta}^{\mathrm{insertion}}=\frac{1}{N_{S}^{\mathrm{II}}+r} \prod_{i=2}^{r} P_{\beta}^{s}(i)
$$

and

$$
\begin{aligned}
P_{\beta}^{\mathrm{Gibbs}}= & \frac{N_{S} !}{\left(N_{S}^{\mathrm{I}}-r\right) !\left(N_{S}^{\mathrm{II}}+r\right) !} \times \frac{M !}{\left(M^{\mathrm{I}}+1\right) !\left(M^{\mathrm{II}}-1\right) !} \\
& \times \exp \left(N^{\mathrm{I}} \ln V^{\mathrm{I}}+N^{\mathrm{II}} \ln V^{\mathrm{II}}-\beta P V^{\mathrm{I}}-\beta P V^{\mathrm{II}}\right. \\
& \left.-\beta U_{\beta}^{\mathrm{II}}-\beta U_{\beta}^{\mathrm{II}}\right) .
\end{aligned}
$$

In order to satisfy the condition of microscopic reversibility, $P_{\alpha \beta}=P_{\beta \alpha}$, the acceptance criterion must obey the following relationship:

$$
P^{S W}=\frac{P_{\alpha \beta}^{a c}}{P_{\beta \alpha}^{a c}}=\frac{M^{\mathrm{II}} N_{S}^{\mathrm{I}}}{\left(M^{\mathrm{I}}+1\right)\left(N_{S}^{\mathrm{II}}+r\right)} \times \frac{W^{\text {old }}}{W^{\text {new }}} \times \exp (-\beta \Delta U) .
$$

In this equation $\Delta U$ is the total energy difference due to the Monte Carlo move, which includes both the bond energy of the polymer chain and the repulsion energy $\left(U=U^{\text {rep }}\right.$ $\left.+U^{b}\right)$. By introducing

$$
\Omega=\frac{W}{\prod_{i=2}^{r} \exp \left(-\beta U^{b}\left(r_{i-1, i}\right)\right)},
$$

we can also write Eq. (A9) as

$$
\begin{aligned}
P^{S W}=\frac{P_{\alpha \beta}^{a c}}{P_{\beta \alpha}^{a c}}= & \frac{M^{\mathrm{II}} N_{S}^{\mathrm{I}}}{\left(M^{\mathrm{I}}+1\right)\left(N_{S}^{\mathrm{II}}+r\right)} \times \frac{\Omega^{\text {new }}}{\Omega^{\text {old }}} \\
& \times \exp \left(-\beta \Delta U^{\text {rep }}\right) .
\end{aligned}
$$

This last equation more clearly shows the similarity with the acceptance criterion used in a conventional CBMC algorithm.

${ }^{1}$ P. J. Hoogerbrugge and J. M. V. A. Koelman, Europhys. Lett. 19, 155 (1992).

${ }^{2}$ J. M. V. A. Koelman and P. J. Hoogerbrugge, Europhys. Lett. 21, 363 (1993).

${ }^{3}$ Y. Kong, C. W. Manke, W. G. Madden, and A. G. Schlijper, Int. J. Thermophys. 15, 1093 (1994)

${ }^{4}$ A. G. Schlijper, P. J. Hoogerbrugge, and C. W. Manke, J. Rheol. 39, 567 (1995)

${ }^{5}$ R. D. Groot and P. Warren, J. Chem. Phys. 107, 4423 (1997).

${ }^{6}$ N. A. Spenley, Europhys. Lett. 49, 534 (2000).

${ }^{7}$ P. Español and P. B. Warren, Europhys. Lett. 30, 191 (1995).

${ }^{8}$ R. D. Groot and T. J. Madden, J. Chem. Phys. 108, 8713 (1998).

${ }^{9}$ R. D. Groot, T. J. Madden, and D. J. Tildesley, J. Chem. Phys. 110, 9739 (1999). 
${ }^{10}$ S. Jury, P. Bladon, M. Cates, S. Krishna, M. Hagen, N. Ruddock, and P. Warren, Phys. Chem. Chem. Phys. 1, 2051 (1999).

${ }^{11}$ M. Venturoli and B. Smit, Phys. Chem. Comm. 10 (1999).

${ }^{12}$ A. Z. Pangiotopoulos, Mol. Phys. 61, 813 (1987).

${ }^{13}$ A. Z. Pangiotopoulos, N. Quirke, M. Stapleton, and D. J. Tildesley, Mol. Phys. 63, 527 (1988).

${ }^{14}$ B. Smit, P. de Smedt, and D. Frenkel, Mol. Phys. 68, 931 (1989).

${ }^{15}$ M. Laso, J. J. de Pablo, and U. W. Suter, J. Chem. Phys. 97, 2817 (1992).

${ }^{16}$ G. C. A. M. Mooij, D. Frenkel, and B. Smit, J. Phys.: Condens. Matter 4, L255 (1992).

${ }^{17}$ S. M. Willemsen, T. J. H. Vlugt, H. C. J. Hoefsloot, and B. Smit, J. Comput. Phys. 147, 507 (1998).

${ }^{18} \mathrm{D}$. Frenkel and B. Smit, Understanding Molecular Simulation. From Algorithms to Applications (Academic, San Diego, 1996).

${ }^{19}$ D. A. Kofke and E. O. Glandt, Mol. Phys. 64, 1105 (1988).

${ }^{20}$ M. R. Stapleton, D. J. Tildesley, and N. Quirke, J. Chem. Phys. 92, 4456
(1990)

${ }^{21}$ M. Müller and N. B. Wilding, Phys. Rev. E 51, 2079 (1995).

${ }^{22}$ T. Dobashi, M. Nakata, and M. Kaneko, J. Chem. Phys. 72, 6685 (1980).

${ }^{23}$ K. Shinozaki, T. van Tan, Y. Saito, and T. Nose, Polymer 23, 278 (1982).

${ }^{24}$ A. D. Mackie, A. Z. Pangiotopoulos, and S. K. Kumar, J. Chem. Phys. 102, 1014 (1995).

${ }^{25}$ N. B. Wilding, M. Muller, and K. Binder, J. Chem. Phys. 105, 802 (1996).

${ }^{26}$ Q. Yan, H. Liu, and Y. Hu, Macromolecules 29, 4066 (1996).

${ }^{27}$ A. Z. Pangiotopoulos, V. Wong, and M. A. Floriano, Macromolecules 31, 912 (1998).

${ }^{28}$ F. A. Escobedo and J. J. de Pablo, Macromolecules 31, 900 (1999).

${ }^{29}$ F. A. Escobedo and J. J. de Pablo, Mol. Phys. 87, 347 (1996).

${ }^{30}$ B. Smit and D. Frenkel, Mol. Phys. 68, 951 (1989).

${ }^{31}$ D. G. Green, G. Jackson, E. de Miguel, and L. F. Rull, J. Chem. Phys. 101, 3190 (1994) 